\title{
Síndrome de Roberts: reporte de un caso
}

\author{
Roberts syndrome: case report
}

\author{
Hugo Xavier Guamán Roldán,* Martín Fabián Díaz Vintimilla*
}

\section{RESUMEN}

El síndrome de Roberts es un trastorno genético descrito por John Roberts en 1919, este síndrome también es conocido como pseudotalidomídico o focomelia (acortamiento severo de extremidades), el cual se caracteriza por anomalías tanto en extremidades como a nivel craneofacial. Reporte de caso: El presente trabajo expone el caso de una paciente de sexo femenino de cinco años de edad, quien presenta secuelas de paladar hendido, ausencia de miembro torácico derecho, hipoplasia de miembro torácico izquierdo, oligodactilia, focomelia, cardiopatía congénita y anomalía dentofacial (laterognatia). Material y métodos: El Servicio de Cirugía Oral y Maxilofacial optó por realizar un procedimiento de distracción osteogénica mandibular (DOM) para corregir la laterognatia, lo cual se consiguió satisfactoriamente. Resultados: Se logró una osteogénesis exitosa, la cual se reflejó en una mejoría de patrones estéticos y funcionales. La DOM es una opción de tratamiento para alteraciones craneofaciales en los pacientes con síndrome de Roberts. Conclusiones: Este tipo de tratamiento debería realizarse a edades tempranas considerando los beneficios que se causan sobre el paciente, tomando en cuenta que serán necesarios otros tratamientos posteriormente.

Palabras clave: Síndrome de Roberts, distracción osteogénica mandibular, focomelia.

\section{ABSTRACT}

Roberts syndrome is a genetic disorder described by John Roberts in 1919 also known as pseudothalidomidic or phocomelia (severe limb shortening) which is characterized by limb and craniofacial abnormalities. Case report: The present paper exposes the case of a female five-year-old patient with cleft palate sequelae, absence of right thoracic limb, left thoracic limb hypoplasia, oligodactyly, focomelia, congenital heart disease and dentofacial anomaly (laterognatia). Material and methods: The Oral and Maxillofacial Surgery service decides to proceed with a mandibular distraction osteogénesis (MDO) process to correct laterognatia which is achieved satisfactorily. Results: Successful osteogenesis is achieved which is reflected in an improvement of aesthetic and functional patterns. Conclusions: This kind of treatment should be carried out at an early age considering the benefits on the patient, considering that other treatments will be necessary later.

Keywords: Roberts syndrome, mandibular distraction osteogenes, phocomelia.

Cirujano Oral y Maxilofacial. Hospital Vicente Corral Moscoso.

‡ Estudiante de la Carrera de Odontología.

Universidad de Cuenca.

Correspondencia:

Dr. Hugo Xavier Guamán Roldán

Cuenca-Ecuador.

E-mail: hxgr@hotmail.com 


\section{INTRODUCCIÓN}

El síndrome de Roberts o síndrome de focomelia es un trastorno autosómico recesivo, fue descrito por primera vez en 1919 por John Roberts en tres hermanos afectados de una pareja italiana de primos en primer grado. Este trastorno es causado por la mutación de ESCO2 encargado de la codificación de proteínas, su alteración conduce a una división celular retardada, a un aumento de la apoptosis y a una proliferación celular. ${ }^{1}$

Alrededor de 150 casos han sido reportados en individuos de diversos orígenes raciales y étnicos, los cuales presentaban un retraso del crecimiento tanto prenatal como postnatal, anomalías craneofaciales, oligodactilia y la reducción bilateral de las extremidades, a nivel facial y oral se ha reportado: hipertelorismo, exoftalmos, labio y paladar hendido bilateral, mala posición dental, laterognatia, macroglosia, rafe palatino medio prominente, hipoplasia del esmalte, macrodoncia, labios inferiores evertidos, entre otros; la morfología de la mandíbula en estos pacientes es diferente en la porción anterior al ángulo de la mandibula, ya que existen longitudes sagitales más cortas, ángulos más pronunciados y una mayor forma elíptica. Existe una prevalencia de esta condición estimada de 0.62 casos por cada 100,000 habitantes. ${ }^{1-5}$

La distracción ósea se define como el proceso mediante el cual se corrigen deformidades esqueletales mediante un proceso de tracción ejercida sobre los huesos. La distracción osteogénica mandibular (DOM) fue presentada por Rosenthal en 1927 al utilizar un aparato dental intraoral que activó gradualmente durante un mes tras obtener resultados exitosos en el tratamiento de pacientes sindrómicos que presentaron discrepancia ósea de 15 hasta 50 $\mathrm{mm} .{ }^{6,7}$ El proceso de distracción ósea tiene como objetivo final la generación de hueso, esto se obtiene mediante un proceso en el cual se respetan los siguientes pasos:

- Se inicia realizando una corticotomía, tomando siempre en cuenta que la preservación del periostio es indispensable para el proceso de osteogénesis, y se procede a colocar el distractor óseo indicado según sea el caso.

- Tras la cirugía se mantiene una fase de fijación neutra de entre cinco a 10 días, de esta manera se permite la formación de un callo óseo y el proceso de microvascularización.

- Transcurrido este tiempo se procede a la distrac- ción de forma gradual de los segmentos del hueso. Estas fuerzas controladas (1 $\mathrm{mm}$ de distracción cada día) estimulan la osteogénesis y a la histogénesis, las cuales permiten la formación de hueso en los segmentos osteotomizados y formación de tejido fibroso en el espacio entre estos segmentos.

- Finalmente se mantiene una fase final de fijación, la cual es de tres a seis meses de duración. ${ }^{8-10}$

El objetivo de la DOM abarca ventajas que mejoran la calidad de vida del paciente tanto en el ámbito estético-dental como del desarrollo e incluso mejora la visión laríngea, la vía aérea, la arquitectura del sueño y la saturación de oxígeno, obteniendo una morfología mandibular y medidas cefalométricas aceptables. ${ }^{3,11}$

Se deben considerar las posibles complicaciones que conlleva este tratamiento. ${ }^{12} \mathrm{Li}$, Gerety, Xu, Bartlett y Taylor (2017) reportaron complicaciones como la avería del dispositivo distractor, siendo necesario su reemplazo cuatro semanas después de la cirugía inicial, de igual manera mencionan la formación de abscesos en las heridas que requirieron intervención quirúrgica; cabe recalcar que estas complicaciones no causaron alteraciones importantes en el tratamiento, por lo que los pacientes culminaron el mismo de forma exitosa. ${ }^{11}$

En la actualidad, este proceso se ve facilitado por software de computadora para una planificación en tres dimensiones y el uso de modelos de estereolitografía para indicar el sitio correcto donde se debe realizar la osteotomía, la fabricación de guías y el premoldeado de placas para prevenir la tracción no uniforme, lo cual brinda resultados más predictivos. ${ }^{13}$

\section{REPORTE DEL CASO}

Paciente de sexo femenino de cinco años de edad acude con sus padres al Hospital Vicente Corral Moscoso debido a que presentaba secuelas de paladar hendido, ausencia de miembro torácico derecho, hipoplasia de miembro torácico izquierdo, oligodactilia, focomelia, cardiopatía congénita y anomalía dentofacial (laterognatia) (Figura 1). El diagnóstico de los médicos del hospital es: síndrome de Roberts. La paciente es derivada al Servicio de Cirugía Oral y Maxilofacial del hospital para ser evaluada y posteriormente intervenida para tratamiento de laterognatia (Figura 2). El cirujano a cargo del servicio planeó realizar una cirugía de DOM con el objetivo de corregir la alteración ósea a nivel de la rama mandibular derecha (Figura 3), la misma que repercute a nivel estético en el 


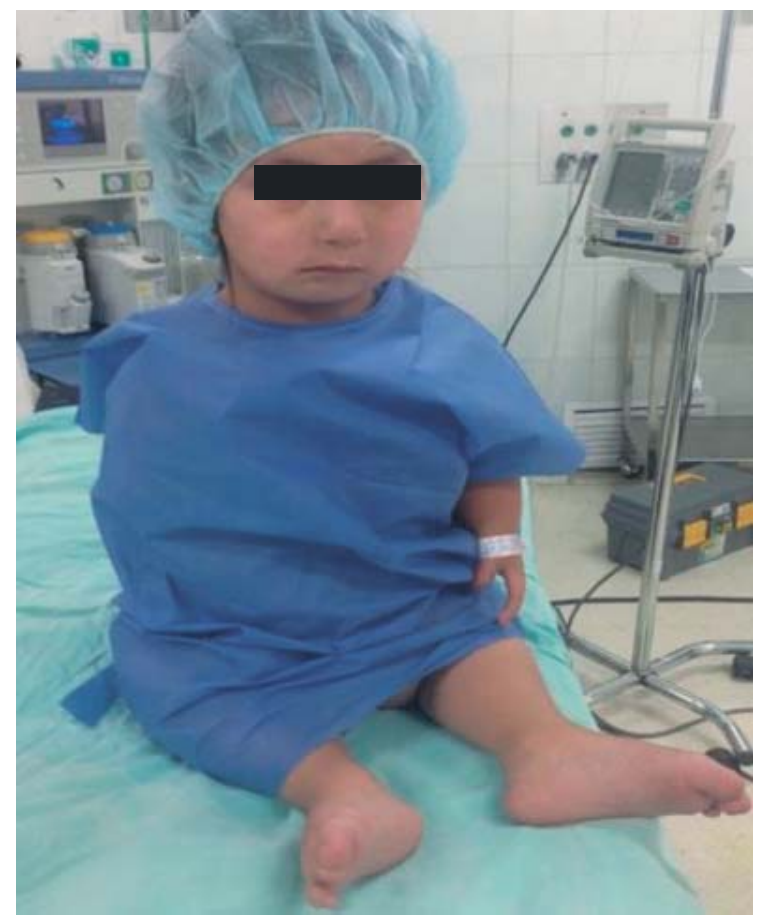

Figura 1: Se observa la ausencia de miembro torácico derecho, hipoplasia de miembro torácico izquierdo, oligodactilia, focomelia y laterognatia.

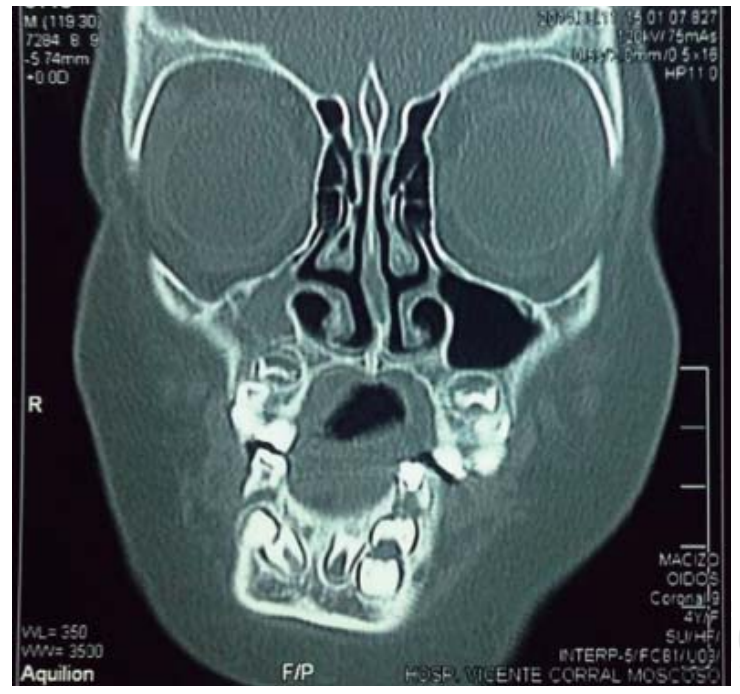

Figura 2: En un corte coronal de la tomografía axial computarizada se puede apreciar la anomalía dentofacial y la desviación mandibular hacia el lado derecho (laterognatia).

rostro de la paciente, quien para compensar esta asimetría inclinaba la cabeza hacia el lado contrario. Se planificó un avance mandibular univectorial de la rama derecha de $12 \mathrm{~mm}$ con la ayuda de un distractor óseo univectorial de traslación A-B ( ${ }^{\circ}$ DePuy Synthes, Switzerland).

\section{MATERIAL Y MÉTODOS}

Bajo anestesia general e intubación orotraqueal se procede a infiltrar el lecho quirúrgico con lidocaína al $2 \%$ con vasoconstrictor al 1:100,000 con fines hemostáticos.

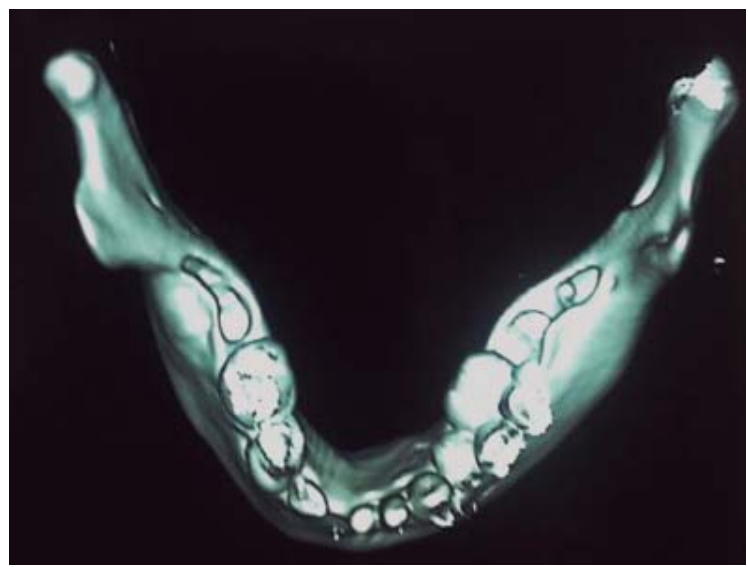

Figura 3: Morfología mandibular asimétrica causada por una alteración en el desarrollo del lado derecho de la misma (micrognatia).

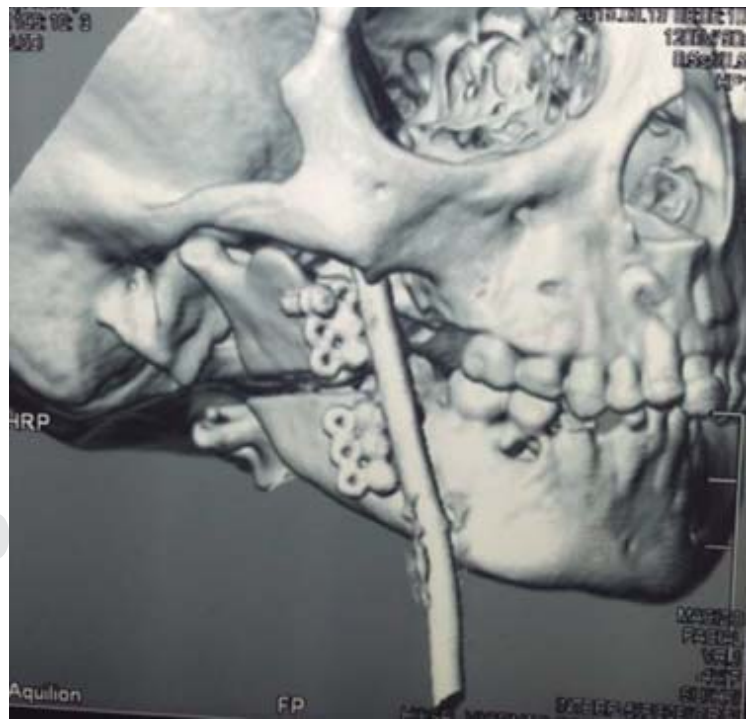

Figura 4: Reconstrucción 3D de la tomografía axial computarizada. Muestra la posición del distractor óseo con respecto a los segmentos óseos separados por un espacio, en el cual se dará un proceso de osteogénesis durante el periodo de fijación neutra. 


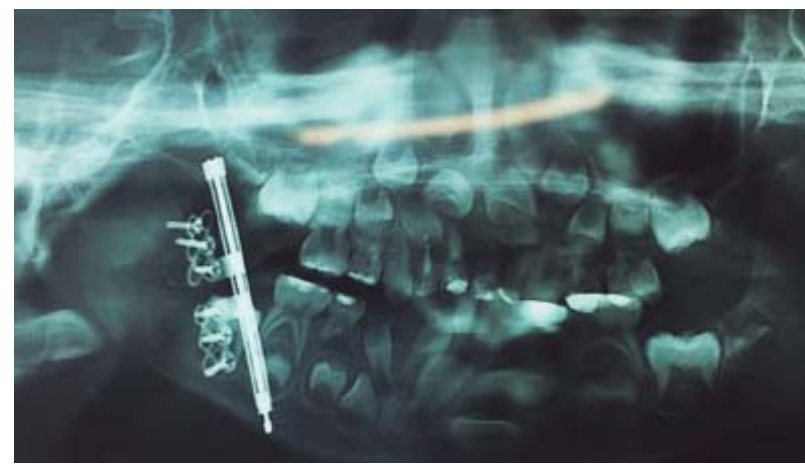

Figura 5: Radiografía panorámica de control realizada previo a la remoción del distractor una vez finalizada la fase final de fijación.

Se inicia con una incisión intraoral a nivel de la rama mandibular con hoja de bisturí No. 15 conjuntamente con electrobisturí se expone cuerpo, ángulo y rama mandibular para marcar el sitio de osteotomía evitando órganos dentarios temporarios y gérmenes de las piezas permanentes con base en la radiografía panorámica.

Se realiza la corticotomía en la rama mandibular del lado derecho por encima de la língula, se termina el corte con un osteótomo fino y se procede a fijar el distractor con seis tornillos monocorticales del sistema 2.0. Se procede a suturar las incisiones por capas con suturas absorbibles.

Se mantiene al distractor inactivo con el brazo extensor flexible expuesto de manera percutánea para permitir la activación, transcurrido un periodo de latencia de cinco días se inicia el proceso de distracción activa de $1 \mathrm{~mm}$ al día durante 12 días utilizando un activador, el cual se gira en sentido antihorario (11/2 giros para $1 \mathrm{~mm}$ ) (Figura 4). Posterior a los 12 días se retira bajo anestesia local el brazo extensor y se inicia el periodo de consolidación.
Después de tres meses el distractor es retirado y se consigue un avance mandibular de $12 \mathrm{~mm}$ en el lado derecho, esto fue comprobado de forma clínica y por medio de tomografías (Figura 5).

\section{RESULTADOS}

Cumpliendo con los objetivos deseados según la planificación, se obtuvo una osteogénesis exitosa al igual que una buena consolidación en la región de la osteotomía, las cuales dieron como resultado una mejoría estética, una relación dental en coordinación de arcada, mejora en la vía aérea y una posición natural de la cabeza (Figura 6).

\section{DISCUSIÓN}

La DOM es el procedimiento mediante el cual estos casos deben ser tratados en pacientes menores a los 14 años para mejorías estéticas temporales, ${ }^{14}$ tomando en cuenta que el tratamiento no finaliza en esta etapa. Los pacientes sometidos a distracción osteogénica deben ser sometidos a seguimientos a largo plazo; además, las futuras cirugías no deben ser descartadas luego de haber alcanzado su punto máximo de crecimiento. Un estudio de Ko, Chen y Lo (2017) demostró que una vez finalizada la distracción, en $90 \%$ de los pacientes la mandíbula continúa con su crecimiento, causando nuevamente asimetrías en un futuro, las cuales deberán ser corregidas con cirugía ortognática. ${ }^{15}$

El motivo por el cual se realiza este tratamiento a edades tempranas es para evitar que el paciente atraviese por eventos psicosociales severos y en caso que fuese necesario un nuevo tratamiento la discrepancia maxilomandibular no sea tan marcada, causando las respectivas consecuencias estéticas. ${ }^{14}$
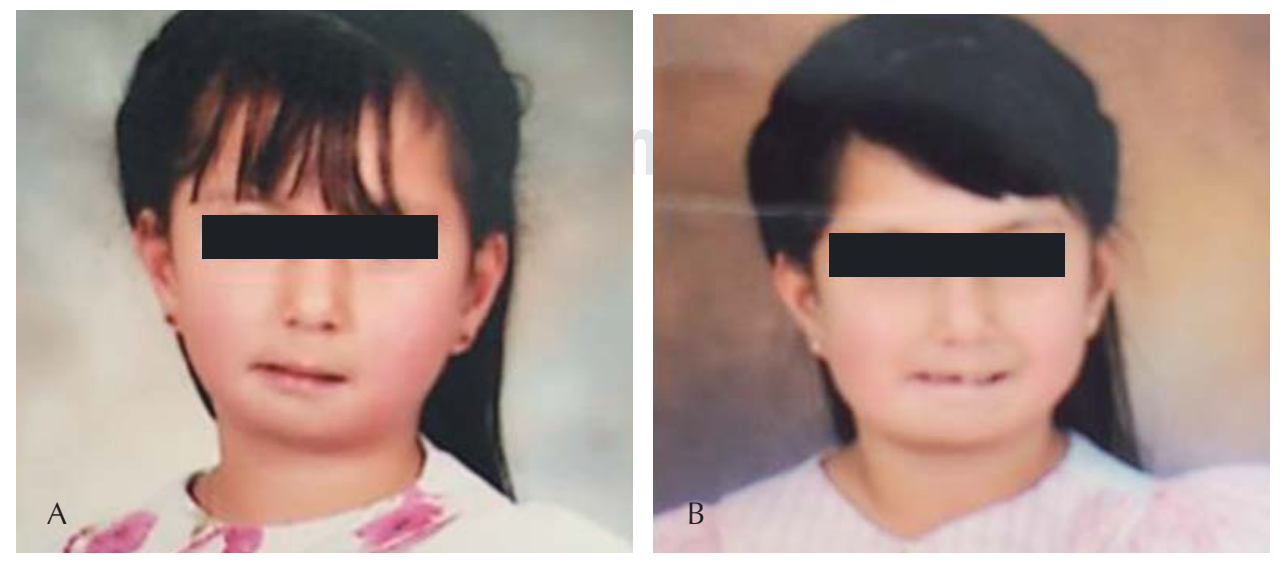

Figura 6:

A) Foto de la paciente antes del tratamiento de distracción osteogénica mandibular (DOM). B) Foto de la paciente tras el avance de $12 \mathrm{~cm}$ de la rama mandibular derecha, se evidencian los resultados estéticos obtenidos en el rostro de la paciente, existe una simetría facial. 


\section{CONCLUSIONES}

1. La DOM es una opción de tratamiento para alteraciones craneofaciales en los pacientes con síndrome de Roberts.

2. El manejo multidisciplinario es vital en este tipo de pacientes.

3. Es importante realizar este tratamiento en edades tempranas, considerando los beneficios psicológicos y del desarrollo que éste puede causar sobre el paciente.

4. Es necesario entender que la distracción ósea es de gran ayuda; sin embargo, es necesario plantear la necesidad de otros tratamientos en un futuro.

\section{BIBLIOGRAFÍA}

1. Ismail S, Essawi M, Sedky N, Helmy N, Fayez A. Roberts syndrome: clinical and cytogenetic studies in 8 Egyptian patients and molecular studies in 4 patients with genotype/phenotype correlation. Genet Couns. 2016; 27: 305-323.

2. Vega H, Waisfisz Q, Gordillo M, Sakai N, Yanagihara I, Yamada $\mathrm{M}$, et al. Roberts syndrome is caused by mutations in ESCO2, a human homolog of yeast ECO1 that is essential for the establishment of sister chromatid cohesion. Nat Genet. 2005; 37 (5): 468-470.

3. Susarla SM, Evans KN, Kapadia H, Vasilakou N, Egbert MA, Hopper RA. Distraction osteogenesis normalizes mandibular body-symphysis morphology in infants with robin sequence. J Oral Maxillofac Surg. 2018; 76 (1): 169-179.
4. Freeman MV, Williams DW, Schimke RN, Temtamy SA, Vachier E, German J. The Roberts syndrome. Clin Genet. 1974; 5 (1): 1-16.

5. Waldenmaier $C$, Aldenhoff $P$, Klemm T. The Roberts' syndrome. Hum Genet. 1978; 40 (3): 345-349.

6. Rossini G, Vinci B, Rizzo R, Pinho TMD, Deregibus A. Mandibular distraction osteogenesis: a systematic review of stability and the effects on hard and soft tissues. Int J Oral Maxillofac Surg. 2016; 45 (11): 1438-1444.

7. Ortiz CA, López NJ. Distracción osteogénica para reconstrucción tridimensional del reborde alveolar atrófico en el segmento anterior maxilar. Rev Mex Cir Bucal Maxilofac. 2018; 15: 57-65.

8. Verlinden CR. Maxillofacial distraction osteogenesis: experiences, results \& dilemmas. 2016.

9. Ransom RC, Leavitt T, Barnes LA, Marshall CD, Wan DC, Longaker MT. A mouse model of mandibular distraction osteogenesis. Plast Reconstr Surg Glob Open. 2017; 5 (4 Suppl): 93-94.

10. Flores GR, Hernández CJ, Muruaga LA. Transporte óseo alveolar en labio y paladar hendido. Rev Mex Cir Bucal Maxilofac. 2016; 12: 57-62.

11. Li J, Gerety PA, Xu W, Bartlett SP, Taylor JA. Cephalometric improvement of severe microretrognathia with an anterior open bite using curvilinear mandibular distraction osteogenesis. J Craniofac Surg. 2017; 28 (5): 1242-1247.

12. Zanaty O, El Metainy S, Abo Alia D, Medra A. Improvement in the airway after mandibular distraction osteogenesis surgery in children with temporomandibular joint ankylosis and mandibular hypoplasia. Pediatr Anesth. 2016; 26 (4): 399-404.

13. Shah S, O'Connor R, Watson J, Srinivasan D, Sidebottom A. Use of three-dimensional printing to assess transport vectors in mandibular distraction osteogenesis. Br J Oral Maxillofac Surg. 2017; 55 (1): 74-76.

14. Polley J, Girotto J, Mann R. 130. Mandibular distraction osteogenesis in hemifacial microsomia: what is appropriate surgical timing? Cleft Palate Craniofac J. 2016; 53 (4): E135.

15. Salcines A, Peacock ZS, Troulis M, Kaban LB. Long-term effects of mandibular distraction osteogenesis. J Oral Maxillofac Surg. 2016; 74 (9): e13. 